\title{
Caracterización florística de ambientes de la cuenca baja del Río Cucurital, afluente del Río Caroní, Estado Bolívar, Guayana Venezolana
}

\author{
Leyda RODRÍGUEZ¹, Giuseppe COLONNELLO²
}

\begin{abstract}
RESUMEN
Como una contribución al conocimiento florístico de la cuenca del Río Caroní se realizaron colecciones botánicas en varios ambientes de la cuenca baja del Río Cucurital, que incluyen formaciones boscosas, tanto en penillanura como ribereñas, morichales, sabanas, arbustales y ambientes perturbados; en los bosques se hicieron colecciones en parcelas de 0,1 ha y fuera de ellas. De manera general, las familias con mayor cantidad de especies fueron Melastomataceae (46), Rubiaceae (33), Euphorbiaceae (23), Cyperaceae (19), Myrtaceae (18), Fabaceae (18), Clusiaceae (15), Arecaceae (15), Caesalpiniaceae (14), Chrysobalanaceae (14), Lauraceae (14), Poaceae (13) y Burseraceae (12), siendo las más representativas de la cuenca baja. Los géneros con mayor cantidad de especies fueron Miconia (17), Psychotria (13), Ocotea (10), Myrcia (8), Protium (7), Licania (7) y Rhynchospora (7). Melastomataceae y Rubiaceae predominaron en todos los ambientes con excepción de la sabana; Lauraceae, Burseraceae, Arecaceae, Chrysobalanaceae y Euphorbiaceae fueron las de mayor importancia en los bosques, y Myrtaceae y Cyperaceae en los bosques ribereños, esta última junto con las Poaceae predominaron en morichal y sabana. Se encontraron 19 especies restringidas a la Guayana venezolana, de las cuales 12 se conocen sólo del estado Bolívar, evidenciando que las condiciones ecológicas, topográficas y edáficas determinan la presencia de elementos florísticos particulares. Las actividades humanas son escasas, lo que se ha reflejado en poca alteración de las comunidades vegetales y en el mantenimiento del carácter prístino de los ambientes. Los escasos ambientes intervenidos han sido colonizados por especies herbáceas y arbustivas típicas de la región.
\end{abstract}

PalabraS Clave: Arbustal, Canaima, Florística, Guayana, Morichal, Río Caroní, Sabana, Venezuela.

\section{Floristic charaterization of enviroments of the lower Cucurital river basin, affluent of Caroní river, Bolívar State, Venezuelan Guayana}

\begin{abstract}
As a contribution to the floristic knowledge of the Caroní river basin, botanical collections were made in several environments of the lower Cucurital river basin, including forests, riverine forests, palm swamp forests (morichal), savanna, scrublands and altered areas. In middle and tall forests additional collections were made in plots of 0.1 ha. In general, the families with the highest number of species were Melastomataceae (46), Rubiaceae (33), Euphorbiaceae (23), Cyperaceae (19), Myrtaceae (18), Fabaceae (18), Clusiaceae (15), Arecaceae (15), Caesalpiniaceae (14), Chrysobalanaceae (14), Lauraceae (14), Poaceae (13) and Burseraceae (12), being the most representative of the low river basin. The genera with highest number of species are Miconia (17), Psychotria (13), Ocotea (10), Myrcia (8), Protium (7), Licania (7) and Rhynchospora (7). Melastomataceae and Rubiaceae families predominated in all environments with exception of the savanna. In the forest, Lauraceae, Burseraceae, Arecaceae, Chrysobalanaceae and Euphorbiaceae were the most abundant, as such as Myrtaceae and Cyperaceae in the riverine forests. Cyperaceae and Poaceae predominated in morichal and savanna. Nineteen species were previously reported only to the Venezuelan Guayana. Twelve of these are only known to the Bolivar state, reinforcing the idea that ecological, topographic and edaphic conditions can determine the presence of particular floristic elements. Human activities are scarce, which is reflected in unaltered vegetal communities and environments. The few altered communities have been colonized by native herbaceous and scrub species.
\end{abstract}

KEYWORDS: Canaima, Caroní river, Floristics, Guiana, Palm swamp forest, Savanna, Scrubland, Venezuela.

\footnotetext{
1 Fundación Instituto Botánico de Venezuela, Herbario Nacional de Venezuela. Apartado Postal 2156, Caracas, 1010A. Venezuela. e-mail: leyda.rodriguez@ucv.ve

${ }^{2}$ Museo de Historia Natural La Salle. e-mail: giuseppe.colonnello@fundacionlasalle.org.ve
} 


\section{INTRODUCCIÓN}

La región de la Guayana venezolana se caracteriza por una alta diversidad de comunidades biológicas de gran riqueza florística y ecológica con altos niveles de endemismo (Berry et al., 1995a; Huber, 1995a). La cuenca del río Caroní es una de las más grandes dentro de la Guayana venezolana, e incluye una serie de afluentes entre los que se encuentra el río Cucurital. Presenta una gama de formaciones vegetales, estrechamente relacionadas con una variada multitud de paisajes geográficos, que abarca desde planicies aluviales y peniplanicies a $50 \mathrm{~m}$ snm hasta peniplanicies disectadas y cumbres inaccesibles a casi $2800 \mathrm{~m}$ (Huber, 1986).

El conocimiento botánico de la cuenca del Caroní se inició en 1755 durante la expedición de Pehr Loefling a la Guayana. Desde entonces se han realizado numerosas exploraciones, que se han intensificado desde 1960 hasta el presente, pudiéndose decir que casi todas las cumbres han sido visitadas con el uso de helicópteros (Huber, 1986, 1995c). La información botánica del conjunto de exploraciones realizadas se ha recopilado en la obra Flora of Venezuelan Guayana (Steyermark et al., 1995-2005).

La cuenca del río Caroní es de gran importancia económica para Venezuela por la construcción de plantas hidroeléctricas (p.e. Guri, Caruachi, Macagua) que aportan gran parte de la energía requerida para el desarrollo del país. Por esta razón, es la cuenca mejor conocida en términos de topografía, clima, hidrografía y vegetación (Huber, 1995b). Si bien en esta cuenca se han realizado numerosas investigaciones botánicas, existen zonas poco conocidas, entre las que se pueden señalar las del suroeste de la cuenca media (Huber, 1986) donde se ubica la cuenca del Río Cucurital. Esto se evidencia también por los escasos reportes de esta área, encontrados en los diferentes volúmenes de la obra "Flora of Venezuelan Guayana". El presente trabajo es una contribución al conocimiento florístico de comunidades poco exploradas de la cuenca del Río Caroní y un aporte a la Flora de la Guayana venezolana.

\section{ÁREA DE ESTUDIO}

La cuenca del río Cucurital se ubica en la vertiente oeste del Auyán-tepui (Figura 1). Se forma predominantemente por los cauces que discurren desde la cima del tepui y en menor grado por afluentes originados por la escorrentía de las tierras bajas. El curso principal se origina por encima de los $1.400 \mathrm{~m}$ snm cerca del extremo sur del macizo del Auyán-tepui y fluye hacia el noroeste descendiendo, primero de forma abrupta hasta aproximadamente la cota de $500 \mathrm{~m}$ y luego de forma escalonada hasta su confluencia con el río Caroní, a unos 320 m (Colonnello, 1999).
La cuenca puede dividirse en tres sectores: a) alto, por encima de los $500 \mathrm{~m}$ de elevación, b) medio entre 380 y 500 $\mathrm{m}, \mathrm{y}$ c) bajo por debajo de $380 \mathrm{~m}$. El estudio se llevó a cabo en la cuenca baja, en el sector conocido como los Raudales

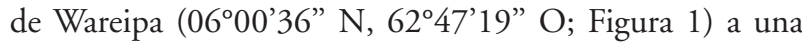
altitud media de $370 \mathrm{~m}$.

En los raudales se intercalan tramos en los que el cauce se halla flanqueado por lajas rocosas, con sectores donde se forma una franja plana de desborde de suelo arenoso que el río inunda estacionalmente y que sirve de hábitat para diversas comunidades de plantas. El macro-relieve de la cuenca baja es mayormente una planicie disectada por los drenajes laterales del río Cucurital, mientras que el meso-relieve en el sector de los raudales consiste en una formación de pequeñas colinas cuyas alturas no sobrepasan los $150 \mathrm{~m}$ por sobre la altiplanicie circundante (Figura 2a). En particular, en la ribera derecha del curso del río se levanta una cresta o pliegue sinclinal que corre longitudinalmente y paralela al Cucurital y separa el curso del mismo de los taludes del Auyán-tepui, mientras que al oeste se halla una altiplanicie ondulada e interrumpida por elevaciones de 40 a $200 \mathrm{~m}$ de altitud, que conforman un sinclinal a lo largo del río Caroní (Figura 2b).

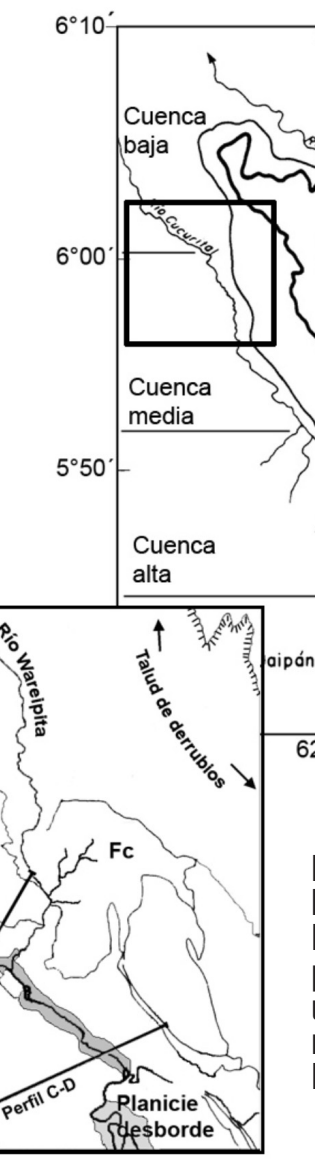


Siguiendo la clasificación de Sioli (1975), las aguas del río Cucurital son negras por la gran cantidad de ácidos húmicos y taninos que han recogido en su recorrido desde su nacimiento, mientras que las aguas río Wareipita, que confluye en el Cucurital en el sector de los raudales, podrían catalogarse como claras, pues debido a su corto recorrido, no contienen gran concentración de ácidos disueltos.

\section{MATERIALES Y MÉTODOS}

Se elaboró un mapa de las principales formaciones vegetales del área estudiada en base a interpretación de las fotografías aéreas $\mathrm{N}^{\circ} 1240,1241,1245$ y 1246 correspondiente a la Misión Nº 050172 de la Dirección de Cartografía Nacional y posterior comprobación en el campo.

Se exploraron varios ambientes y se colectaron muestras botánicas, tanto en época de sequía como en lluvias. Los ambientes incluyen bosques medios en terrenos pendientes (frente de cuesta) que se ubican en la margen derecha del río, bosques altos en peniplanicie, en dirección sur-este y arbustales sobre arena. Así mismo se exploraron el bosque ribereño del río Cucurital (río abajo de los raudales de Wareipa), el bosque ribereño del río Wareipita (aproximadamente a $4 \mathrm{~km}$ de la desembocadura en el río Cucurital), un morichal, varios parches de sabana y lugares intervenidos. En los bosques se realizaron colecciones en dos parcelas de 0,1 ha en los bosques medio y alto, con el objeto de realizar comparaciones cuantitativas con otros bosques de la región.

Las colecciones se realizaron en recorridos a pie en tierra, y en bote a lo largo de las orillas del río. Para cada especie se tomaron hasta cinco (5) muestras. Se hicieron identificaciones preliminares en el campo hasta la categoría de familia y/o género. Las muestras luego de prensadas, fueron preservadas en alcohol isopropílico al $70 \%$, y después se secaron a una temperatura entre $60-70^{\circ} \mathrm{C}$ en una estufa adecuada para tal fin. Posteriormente, fueron identificadas hasta la categoría de especie, mediante el apoyo de la bibliografía existente y la comparación con ejemplares depositados en el Herbario Nacional de Venezuela. Muestras de Pteridófitas, y algunas familias de Angiospermas, como Lauraceae, Myrtaceae, Orchidaceae y Poaceae fueron enviadas a especialistas para su identificación. Las muestras están depositadas en los herbarios VEN, CAR y GUYN.

\section{RESULTADOS Y DISCUSIÓN}

En la cuenca baja del Río Cucurital se encuentra una variada gama de tipos de vegetación (Figura 2), cuya composición florística se describe a continuación:

\section{BOSQUES}

En el sector estudiado de la cuenca del Río Cucurital se pueden encontrar bosques influenciados directamente por el cauce del río (ribereños) y aquellos más alejados de las corrientes, que no lo están.

\section{1.- BOSQUES RIBEREÑOS Y VEGETACIÓN ASOCIADA}

En vegetación ribereña se colectaron en total 52 familias, 90 géneros y 109 especies de plantas vasculares (Tabla 1). Las familias más abundantes son Rubiaceae $(9$ especies), Melastomataceae (8), Myrtaceae (7), Cyperaceae (7), Caesalpiniaceae y Fabaceae (5). Los géneros más representativos son Psychotria, Miconia, Macrolobium y Myrcia. Esta vegetación que acompaña el cauce del río, tiene entre 10 y $15 \mathrm{~m}$ de alto, mostrando un estrato arbustivo intermedio. Está formada por especies leñosas que incluyen árboles, arbustos y lianas, así como por especies herbáceas. Estas últimas crecen en sustrato arenoso formando grupos en la orilla, destacando especies del género Rycnchospora. Entre las especies leñosas que se encuentran en la ribera del Río Cucurital (aguas oscuras) se destacan Tabebuia insignis, Protium trifoliolatum, P. crassipetalum, Macrolobium acaciifolium, M. bifolium, M. multijugum, Peltogyne venosa, Acosmium nitens, Spirotropis longiflora, Ocotea schomburgkiana, Anthodiscus mazarunensis, Croton cuneatus, Parkia pendula y Duroia sprucei, entre otras (Tabla 1). Panopsis rubescens es abundante en algunos sectores hacia de la desembocadura del río en el Caroní. También se detectaron especies trepadoras, como Gnetum leyboldii, Phygranocidia corymbosa, Tetrapterys styloptera y Dilkea acuminata. En lugares donde la sabana alcanza las inmediaciones del río, en la ribera crecen abundantes arbustos, que pueden desarrollarse sobre sustrato rocoso. En sectores donde el río se explaya se pueden observar elementos leñosos en afloraciones rocosas en el cauce del río, tales como Spongioperma cataractarum, Psidium sprucei y Duroia sprucei.
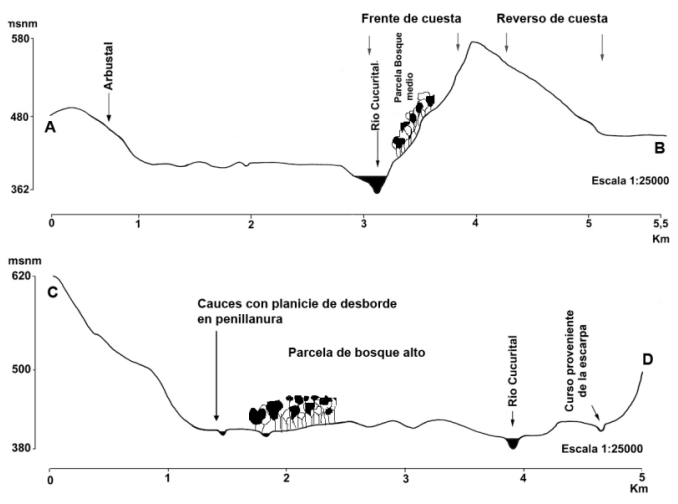

Figura 2 - Perfiles del relieve del sector estudiado en la cuenca baja del Río Cucurital. Ubicación aproximada de las parcelas realizadas en los bosques. 
Tabla 1 - Especies de vegetación ribereña de la cuenca baja del Río Cucurital

\begin{tabular}{|c|c|c|c|}
\hline Familia & Especie & Hábito & Ambiente \\
\hline \multirow[t]{2}{*}{ Acanthaceae } & Justicia calycina (Nees) V.A.W.Graham & Sufrútice & $\mathrm{BR}$, aguas claras \\
\hline & Justicia kunhardtii Leonard & Sufrútice & $\mathrm{BR}$, aguas oscuras \\
\hline Annonaceae & Guatteria cardoniana R.E.Fr. & Árbol & $\begin{array}{l}\text { Vegetación ribereña, abierta, sustrato } \\
\text { rocoso }\end{array}$ \\
\hline \multirow[t]{2}{*}{ Apocynaceae } & Mandevilla scabra (Hoffmanns ex Roem. \& Schult) K.Schum & Trepadora & $\mathrm{BR}$, aguas oscuras \\
\hline & Spongiosperma cataractarum Zarucchi & Arbusto & En lecho de río de aguas oscuras \\
\hline Aquifoliaceae & Ilex jenmanii Loes & Arbusto & $\begin{array}{l}\text { Vegetación ribereña, aguas claras y } \\
\text { oscuras }\end{array}$ \\
\hline Araceae & Urospatha sagittifolia (Rudge) Schott & Hierba & Borde anegado de ríos \\
\hline Arecaceae & Desmoncus polyacanthos Mart. & Palma trepadora & $B R$, aguas oscuras \\
\hline Asteraceae & Calea divaricata Benth. & Arbusto & Vegetación ribereña que no se inunda \\
\hline \multirow[t]{4}{*}{ Bignoniaceae } & Anemopaegma alatum A.H.Gentry & Liana & $\mathrm{BR}$, aguas oscuras \\
\hline & Cydista aequinoctialis (L.) Miers & Liana & BR de aguas oscuras \\
\hline & Phryganocydia corymbosa (Vent.) Bail. & Liana & $\mathrm{BR}$, aguas oscuras \\
\hline & Tabebuia insignis (Miq.) Sandwith & Árbol & $\mathrm{BR}$, aguas claras, lugares rocosos \\
\hline Bombacaceae & Pachira minor (Sims) Hemsl. & Árbol & BR, aguas oscuras \\
\hline Bromeliaceae & Pitcairnia maidifolia (E.Morren) Decne ex Planch. \& Lind. & Hierba & Vegetación ribereña, bajo sombra \\
\hline \multirow{3}{*}{ Burseraceae } & Protium trifoliolatum Engl. & Árbol & $\mathrm{BR}$, aguas oscuras \\
\hline & Protium crassipetalum Cuatrec. & Árbol & BR aguas oscuras \\
\hline & Trattinnickia burserifolia Mart. & Árbol & $\mathrm{BR}$, aguas oscuras \\
\hline \multirow[t]{5}{*}{ Caesalpiniaceae } & Eperua venosa R.S.Cowan & Árbol & $\mathrm{BR}$, aguas claras \\
\hline & Macrolobium acaciifolium (Benth.) Benth. & Árbol & BR, aguas oscuras \\
\hline & Macrolobium bifolium (Aubl.) Pers. & Árbol & $\mathrm{BR}$, aguas claras \\
\hline & Macrolobium multijugum (DC.) Benth. & Árbol & $\mathrm{BR}$, aguas oscuras \\
\hline & Peltogyne venosa (Vahl) Benth. & Árbol & BR, aguas oscuras \\
\hline Caryocaraceae & Anthodiscus mazarunensis Gilly & Árbol pequeño & $\mathrm{BR}$, aguas oscuras \\
\hline \multirow{3}{*}{ Chrysobalanaceae } & Hirtella racemosa Lam. & Árbol & BR, aguas oscuras \\
\hline & Licania parviflora Benth. & Árbol & BR, aguas oscuras \\
\hline & Licania boyanii Tutin & Árbol & BR, aguas oscuras \\
\hline Clusiaceae & Clusia schomburgkiana (Planch. \& Triana) Benth. ex Engl. & Árbol & $\begin{array}{l}\text { Vegetación ribereña, abierta y rocosa, } \\
\text { aguas oscuras }\end{array}$ \\
\hline Connaraceae & Connarus lambertii (DC.) Sagot & Trepadora & BR, aguas oscuras \\
\hline Costaceae & Costus spiralis (Jacq.) Roscoe & Hierba & Vegetación ribereña de aguas claras \\
\hline \multirow[t]{7}{*}{ Cyperaceae } & Hypolytrum pulchrum (Rudge) H.Pfeiff. & Hierba & Vegetación ribereña \\
\hline & Lagenocarpus rigidus Ness & Hierba & Playa arenosa inundable \\
\hline & Rynchospora cephalotes (L.) Vahl & Hierba & Borde de riachuelo de aguas blancas \\
\hline & Rhynchospora pubera (Vahl) Gale & Hierba & BR \\
\hline & Rynchospora unisetosa T.Koyama & Hierba & Vegetación ribereña \\
\hline & Scleria cyperina Kunth & Hierba & Vegetación ribereña, rala, aguas oscuras \\
\hline & Scleria microcarpa Nees & Hierba & En borde anegado de río \\
\hline Cyrillaceae & Cyrilla racemiflora L. & Arbusto & $\begin{array}{l}\text { Vegetación ribereña, aguas oscuras, } \\
\text { sustrato rocoso }\end{array}$ \\
\hline \multirow[t]{4}{*}{ Euphorbiaceae } & Chaetocarpus schomburgkianus (Kuntze) Pax \& Hoffm. & Árbol & Vegetación ribereña, en playa arenosa \\
\hline & Croton cuneatus Klotzsch & Árbol & BR \\
\hline & Pera descipiens (Müll.Arg.) Müll.Arg. & Árbol & BR que no sufre inundación \\
\hline & Pera glabrata (Schott) Baill. & Árbol & BR que no sufre inundación \\
\hline \multirow[t]{5}{*}{ Fabaceae } & Acosmium nitens (Vogel) Yakovlev & Árbol & BR, aguas oscuras \\
\hline & Dalbergia foliosa (Benth.) A.M.Carvalho & Árbol & $\mathrm{BR}$, aguas oscuras \\
\hline & Ormosia bolivarensis (Rudd) Stirton & Árbol & $\mathrm{BR}$, aguas oscuras \\
\hline & Spirotropis longifolia (DC.) Baill. & Árbol & $\mathrm{BR}$, aguas oscuras \\
\hline & Swartzia steyermarkii R.S.Cowan & Árbol & BR, aguas claras \\
\hline
\end{tabular}


Tabla 1 - Continuación

\begin{tabular}{|c|c|c|c|}
\hline Familia & Especie & Hábito & Ambiente \\
\hline \multirow[t]{3}{*}{ Gentianaceae } & Chelonanthus alatus (Aubl.) Pulle & Sufrútice & Vegetación ribereña \\
\hline & $\begin{array}{l}\text { Chelonanthus purpurascens (Aubl.) L. Struwe, S.Nilsson \& } \\
\text { V.A.Albert }\end{array}$ & Hierba & Vegetación ribereña, aguas claras \\
\hline & Tapeinostemon spenneroides Benth. & Hierba & BR, agues oscuras \\
\hline Gesneriaceae & Codonanthe crassifolia (Focke) Morton & Trepadora epífita & $\mathrm{BR}$ \\
\hline Gnetaceae & Gnetum leyboldii Tul. & Liana & $\mathrm{BR}$, aguas oscuras \\
\hline \multirow[t]{2}{*}{ Humiriaceae } & Humiria balsamifera Aubl. & Árbol & $\mathrm{BR}$ \\
\hline & Sacoglottis guianensis Benth. & Árbol & $\mathrm{BR}$ \\
\hline Lauraceae & Ocotea schomburgkiana (Nees) Mez & Árbol & BR, aguas oscuras \\
\hline Lentibulariaceae & Utricularia aff. pusilla Vahl & Hierba & Vegetación ribereña, lugares anegados \\
\hline Loganiaceae & Bonyunia minor N.E.Br. & Árbol pequeño & BR que no sufre inundación \\
\hline Malvaceae & Peltaea surumuensis (Ulbr.) Krapov. \& Cristóbal & Arbusto & Vegetación ribereña inundable \\
\hline \multirow[t]{2}{*}{ Malpighiaceae } & Tetrapteris fimbripetala Adr.Juss. & Liana & $\mathrm{BR}$ \\
\hline & Tetrapteris styloptera Adr.Juss. & Liana & $\mathrm{BR}$, aguas oscuras \\
\hline \multirow[t]{8}{*}{ Melastomataceae } & Adelobotrys permixta Wurdack & Trepadora & Parte interna del BR \\
\hline & Comolia villosa (Aubl.) Triana & Hierba & $\begin{array}{l}\text { Vegetación ribereña, rala sustrato rocoso, } \\
\text { aguas oscuras }\end{array}$ \\
\hline & Henriettea stellaris Berg ex Triana & Árbol & BR de río de aguas claras \\
\hline & Maieta guianensis Aubl. & Arbusto & Vegetación ribereña \\
\hline & Meriania urceolata Triana & Árbol & BR de aguas claras \\
\hline & Miconia ciliata (L.C.Rich.) DC. & Arbusto & Vegetación ribereña, aguas claras \\
\hline & Miconia myriantha Benth. & Árbol & BR de aguas oscuras \\
\hline & Miconia phaeophylla Triana & Árbol & $\begin{array}{l}\text { Vegetación ribereña, sustrato rocoso, } \\
\text { aguas oscuras }\end{array}$ \\
\hline Menispermaceae & Abuta pahnii (Mart.) Krukoff \& Barneby & Liana & BR de aguas oscuras \\
\hline \multirow[t]{3}{*}{ Mimosaceae } & Abarema jupumba (Willd.) Britton \& Killip & Árbol & $\mathrm{BR}$, sustrato rocoso aguas claras \\
\hline & Inga sertulifera (Miq.) Sandwith & Árbol & BR, sustrato rocoso aguas claras \\
\hline & Parkia pendula Benth. ex Walp. & Árbol & $\mathrm{BR}$ \\
\hline Myrsinaceae & Cybianthus spicatus (Kunth) G.Agostini & Árbol pequeño & BR, aguas oscuras \\
\hline \multirow[t]{7}{*}{ Myrtaceae } & Eugenia punicifolia (Kunth) DC. & Arbusto & $\begin{array}{l}\text { Vegetación ribereña rala y con sustrato } \\
\text { rocoso }\end{array}$ \\
\hline & Eugenia pubescens (Kunth) DC. & Arbusto & BR de aguas oscuras \\
\hline & Myrcia fallax (Rich.) DC. & Árbol & $\mathrm{BR}$, aguas oscuras \\
\hline & Myrcia platycada DC. & Arbusto & $\mathrm{BR}$, aguas oscuras \\
\hline & Myrcia pyrifolia (Desv. ex Ham.) Nied. & Árbol & $\mathrm{BR}$, aguas oscuras \\
\hline & Myrciaria tenella (DC.) 0.Berg & Sufrútice & $\mathrm{BR}$, aguas oscuras \\
\hline & Psidium acutangulum DC. & Árbol pequeño & Lecho de río, aguas oscuras \\
\hline Ochnaceae & Sauvagesia ramosissima Spruce & Sufrútice & Vegetación ribereña \\
\hline \multirow[t]{4}{*}{ Orchidaceae } & Cattleya lawrenceana Rchb.f. & Hierba epífita & $\mathrm{BR}$, aguas claras \\
\hline & Polystachya amazonica Schtr. & Hierba epífita & $\mathrm{BR}$, aguas claras \\
\hline & Polystachya aff. foliosa (Hook.) Rchb.f. & Hierba epífita & $\mathrm{BR}$, aguas claras \\
\hline & Scaphyglottis graminifolia (Ruiz \& Pav.) Poepp. \& Endl. & Hierba epífita & $\mathrm{BR}$, aguas claras \\
\hline Passifloraceae & Dilkea acuminata Mast. & Liana & $\mathrm{BR}$, aguas oscuras \\
\hline Polygalaceae & Securidaca marginata Benth. & Liana & BR, aguas oscuras; morichal \\
\hline Poaceae & Raddiella esembeckii (Steud.) Calderón \& Sordestron & Hierba & Vegetación ribereña de aguas claras \\
\hline Proteaceae & Panopsis rubescens (Pohl.) Rusby & Árbol & $\mathrm{BR}$, aguas oscuras \\
\hline Rhizophoraceae & Cassipourea guianensis Aubl. & Árbol & BR \\
\hline \multirow[t]{2}{*}{ Rubiaceae } & Borreria capitata (Ruiz \& Pav.) DC. & Sufrútice & $\begin{array}{l}\text { Vegetación ribereña, arenosa, en lugar } \\
\text { abierto }\end{array}$ \\
\hline & Borreria latifolia (Aubl.) Schum. & Sufrútice & $\begin{array}{l}\text { Vegetación ribereña, arenosa, en lugar } \\
\text { abierto }\end{array}$ \\
\hline
\end{tabular}


Tabla 1 - Continuación

\begin{tabular}{llll}
\hline Familia & Especie & Hábito & Ambiente \\
\hline & Duroia sprucei Rusby & Árbol & BR de aguas oscuras \\
& Palicourea crocea (Sw.) Roem. \& Schult. & Arbusto & BR (corredor) \\
& Psychotria anceps Kunth & Arbusto & BR, aguas oscuras \\
& Psychotria bracteocardia (A.DC.) Müll.Arg. & Arbusto & BR, aguas claras \\
& Psychotria deflexa DC. & Arbusto & BR, no inundable, aguas oscuras \\
& Psychotria hoffmannseggiana (Willd. ex Roem. \& Schult.) Müll. & Arbusto & Boque ribereño \\
\hline Arg. & Psychotria phaneroloma Standl. \& Steyerm. & Arbusto & BR no inundable, aguas oscuras \\
\hline Smilacaceae & Smilax chimantensis Steyerm. \& Maguire & Trepadora & BR, aguas claras \\
\hline Solanaceae & Hawkesiophyton ulei (Dammer) Hunz. & Liana epífita & BR (interior) \\
\hline Theaceae & Archytaea triflora Mart. & Árbol & Vegetación ribereña rala, sustrato rocoso, \\
\hline Thurniaceae & Thurnia sphaerocephala Hook.f. & Hierba & Ambiente inundado, aguas claras \\
Trigoniaceae & Trigonia sericea Kunth & Trepadora & BR, aguas oscuras \\
\hline Turneraceae & Turnera acuta Willd. & Arbusto & Vegetación ribereña, abierta y con \\
Viscaceae & Phoradendron crassifolium (DC.) Eichler & sustrato rocoso \\
\hline Vochysiaceae & Phoradendron aff. undulatum (Pohl.) Eichler & BR, aguas oscuras \\
\hline Xyridaceae & Ruizterania ferruginea (Steyerm.) Marc.-Berti & Parásita & BR, aguas oscuras \\
\hline BR: & Abolboda grandis Griseb. & Árbol & Vegetación ribereña, abierta y con \\
\end{tabular}

BR: Bosque ribereño

En el borde del Río Wareipita (aguas claras) abundan Archytaea triflora, Eperua venosa, Meriania urceolata, Inga sertulifera, Tabebuia insignis y Henrietea stellaris. Entre la vegetación arbustiva y herbácea destacan Miconia ciliata, Justicia calicina, Hypolytrum pulchrum y Thurnia sphaerocephala. En algunos lugares húmedos y sombreados se observaron epífitas como Polystachya amazonica, P. foliosa, Cattleya lawrenceana y Pitcairnia maidifolia, así como la trepadora Smilax chimantensis.

En muchos lugares de la ribera del río Cucurital el bosque ribereño es más abierto y ralo y se observa abundancia de rocas, encontrándose árboles y arbustos dispersos como Ruizterania ferruginea, Clusia schomburgkiana, Cyrilla racemiflora y Eupronia guianensis.

\section{2.- BOSQUES NO RIBEREÑOS}

Estas comunidades se pueden diferenciar en bosques altos, medios y bajos (Figura 3). En total en los bosques altos y medios se inventariaron 64 familias, 143 géneros y 210 especies de plantas vasculares (Tabla 2). Las familias que dominan la flora de estos bosques son Rubiaceae, Melastomataceae, Lauraceae, Burseraceae, Arecaceae, Chrysobalanaceae y Euphorbiaceae, mientras que los géneros más representativos son Psychotria (10 especies), Miconia (8), Licania y Ocotea (6), Protium, Pourouma y Tovomita (5).

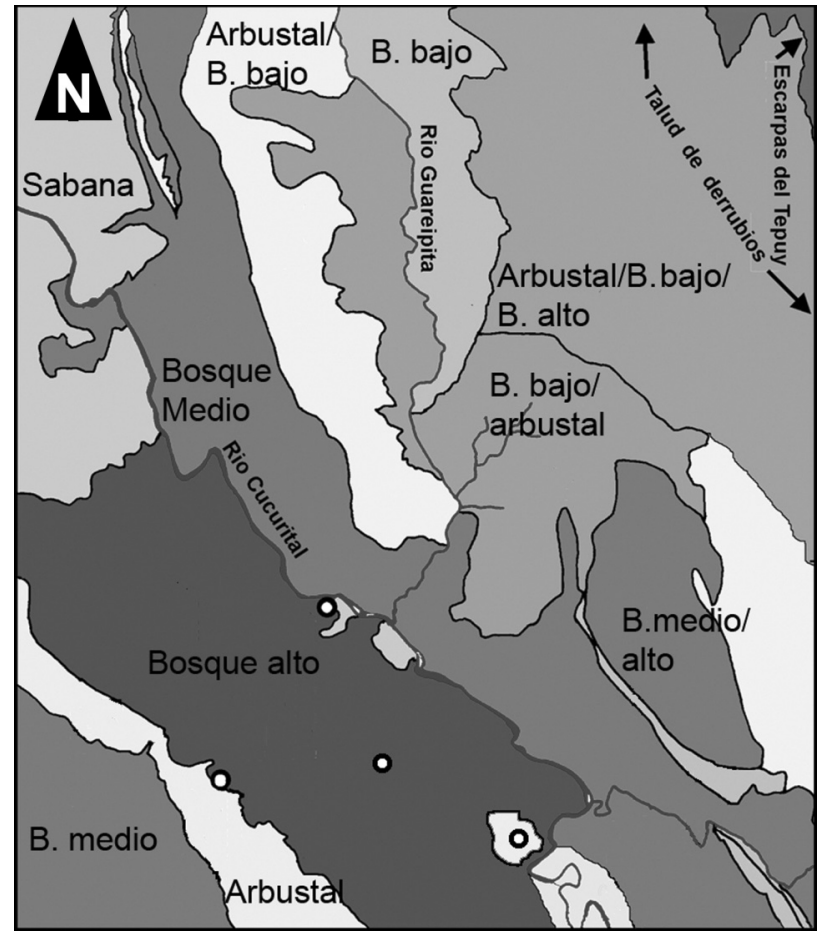

Figura 3 - Distribución de los principales tipos de vegetación encontrados en el sector estudiado de la cuenca baja del Río Cucurital 
Tabla 2 - Especies presentes en los bosques de la cuenca baja del Río Cucurital

\begin{tabular}{|c|c|c|c|}
\hline Familia & Especie & Hábito & Ambiente \\
\hline \multirow[t]{3}{*}{ Apocynaceae } & Aspidosperma exselsum Benth. & Árbol & BAP \\
\hline & Macoubea guianensis Aubl. & Árbol & BMP \\
\hline & Rhigospira quadrangularis (Müll.Arg.) Miers & Árbol & BMP, BAP \\
\hline \multirow[t]{2}{*}{ Anacardiaceae } & Tapirira guianensis Aubl. & Árbol & BMP \\
\hline & Thyrsodium spruceanum Benth. & Árbol & BMP \\
\hline \multirow[t]{6}{*}{ Annonaceae } & Anaxagorea dolichocarpa Sprague \& Sandwith & Árbol & BAP \\
\hline & Duguetia pauciflora Rusby & Árbol & BMP \\
\hline & Duguetia pycnastera Sandwith & Árbol & BAP \\
\hline & Bocageopsis multiflora (Mart.) R.E.Fr. & Árbol & BMP, BAP \\
\hline & Guatteria cardoniana R.E.Fr. & Árbol & BMP \\
\hline & Guatteria ovalifolia R.E.Fr. & Árbol & BMP \\
\hline \multirow[t]{6}{*}{ Araceae } & Anthurium clavigerum Poepp. & Epífita & BAP, en lugar sombreado \\
\hline & Anthurium guayanum G.S.Bunting & Hierba terrestre & $\begin{array}{l}\text { BB en sustrato arenoso y } \\
\text { rocoso }\end{array}$ \\
\hline & Anthurium gracile (Rudge) Schott & Epífita & BAP \\
\hline & Heteropsis flexuosa (Kunth) G.S.Bunting & Trepadora & BMP \\
\hline & Philodendron pedatum (Hook.) Kunth & Epífita & BMP \\
\hline & Philodendron fragantissimum G.S.Bunting & Epífita & BAP \\
\hline Araliaceae & Dendropanax arboreus (L.) Decne \& Planch. & Árbol & BAP \\
\hline \multirow[t]{9}{*}{ Arecaceae } & Astrocaryum gynacanthum Mart. & Palma arborescente & BMP \\
\hline & Attalea maripa Mart. & Palma arborescente & BMP, BAP \\
\hline & Bactris simplicifrons Mart. & Hierba & BMP \\
\hline & Euterpe pracatoria Mart. var. precatoria & Palma arborescente & BMP, BAP \\
\hline & Geonoma deversa (Poit.) Kunth & Palma arborescente & BMP \\
\hline & Geonoma macrostachys Mart. var. poiteauana (Kunth) A.Hend. & Hierba & BAP \\
\hline & Geonoma maxima (Poit.) Kunth & Palma arborescente & BMP \\
\hline & Iriartella setigera (Mart.) H. Wendl. & Palma arborescente & BAP \\
\hline & Oenocarpus bacaba Mart. & Palma arborescente & BMP, BAP \\
\hline \multirow[t]{3}{*}{ Bignoniaceae } & Arrabidaea nigrescens Sandwith & Liana & BMP \\
\hline & Jacaranda copaia (Aubl.) D. Don & Árbol & BAP \\
\hline & Tabebuia subtilis Sprague \& Sandwith & Árbol & BMP \\
\hline \multirow[t]{3}{*}{ Boraginaceae } & Cordia bicolor A.DC. & Árbol & BAP \\
\hline & Cordia aff. exaltata Lam. & Árbol & BAP \\
\hline & Cordia nodosa Lam. & Árbol & BAP \\
\hline Bromeliaceae & Ananas parguazensis Camargo \& L.B.Sm. & Hierba & $\begin{array}{l}\text { BM, en lugares ralos y } \\
\text { sombreados }\end{array}$ \\
\hline \multirow[t]{2}{*}{ Burmanniaceae } & Gymnosiphon cymosus (Benth.) Benth. \& Hook. f. & Hierba saprófita & $\begin{array}{l}\text { BMP, en lugar húmedo y } \\
\text { sombreado }\end{array}$ \\
\hline & Gymnosiphon divaricatus (Benth.) Benth. \& Hook. f. & Hierba saprófita & $\begin{array}{l}\text { BMP, en lugar húmedo y } \\
\text { sombreado }\end{array}$ \\
\hline \multirow[t]{10}{*}{ Burseraceae } & Crepidospermum rhoifolium (Benth.) Triana \& Planch. & Árbol & BAP \\
\hline & Dacryodes chimantensis Steyerm. \& Maguire & Árbol & BMP, BAP \\
\hline & Dacryodes nitens Cuatrec. & Árbol & BAP \\
\hline & Protium aracouchini (Aubl.) Marchand & Árbol & BMP \\
\hline & Protium opacum Swart & Árbol & BMP, BAP \\
\hline & Protium sagotianum Marchand & Árbol & BAP \\
\hline & Protium spruceanum (Benth.) Engl. & Árbol & BMP \\
\hline & Protium trifoliolatum Engl. & Árbol & BMP \\
\hline & Trattinnickia burserifolia Mart. & Árbol & BMP \\
\hline & Trattinnickia lawrancei Standl. ex Swart & Árbol & BMP, BAP \\
\hline \multirow[t]{2}{*}{ Caesalpiniaceae } & Bauhinia guianensis Aubl. & Liana & BAP \\
\hline & Bauhinia rutilans Spruce ex Benth. & Liana & BAP \\
\hline
\end{tabular}


Tabla 2 - Continuación

\begin{tabular}{|c|c|c|c|}
\hline Familia & Especie & Hábito & Ambiente \\
\hline & Eperua jenmanii Oliv. & Árbol & BAP \\
\hline & Tachigali guianensis (Benth.) Zarucchi \& Herend & Árbol & BMP \\
\hline & Tachigali rusby Harms & Árbol & BMP \\
\hline Caryocaraceae & Caryocar pallidum A.C.Sm. & Árbol & BMP \\
\hline \multirow[t]{4}{*}{ Cecropiaceae } & Pourouma guianensis Aubl. & Arbol & BMP \\
\hline & Pourouma bicolor Mart. & Árbol & BMP \\
\hline & Pourouma minor Benoist & Árbol & BAP \\
\hline & Pourouma melinonii Benoist & Árbol & BAP \\
\hline Celastraceae & Goupia glabra Aubl. & Árbol & BMP, BAP \\
\hline \multirow[t]{9}{*}{ Chrysobalanaceae } & Couepia guianensis Aubl. & Árbol & BAP \\
\hline & Hirtella bicornis Mart. \& Zucc. & Árbol & BMP, BAP \\
\hline & Hirtella silicea Griseb. & Árbol & BMP \\
\hline & Licania boyanii Benoist & Árbol & BMP \\
\hline & Licania canescens Benoist & Árbol & BMP \\
\hline & Licania intrapetiolaris Spruce ex Hook. & Árbol & BMP \\
\hline & Licania octandra (Hoffmanns. ex Roem. \& Schult.) Kuntze & Árbol & BMP \\
\hline & Licania steyermarkii Maguire & Árbol & BMP \\
\hline & Licania octandra Griseb. & Árbol & BMP \\
\hline \multirow[t]{7}{*}{ Clusiaceae } & Caraipa tereticaulis Tul. & Árbol & BMP \\
\hline & Caraipa densifolia Mart. & Árbol & BAP \\
\hline & Caraipa richardiana Cambess. & Árbol & BAP \\
\hline & Tovomita eggersii Vesque & Árbol & BAP \\
\hline & Tovomita gracilipes Planch. ex Triana & Árbol & BAP \\
\hline & Tovomita schomburgkii Planch. \& Triana & Árbol & BAP \\
\hline & Tovomita tenuiflora Benth. ex Planch. ex Triana & Árbol & BMP \\
\hline Convolvulaceae & Maripa scandens Aubl. & Liana & BAP \\
\hline Cucurbitaceae & Gurania bignoniacea (Poepp. \& Endl.) C.Jeffrey & Trepadora & BAP \\
\hline \multirow[t]{3}{*}{ Cyperaceae } & Calyptrocarya glomerata (Brongn.) Urb. & Hierba & BMP \\
\hline & Calyptrocarya bicolor (H.Pfeiff.) T.Koyama & Hierba & BAP \\
\hline & Diplasia karataefolia L.C.Rich. & Hierba & BMP \\
\hline Dryopteridaceae & Elaphoglossum glabellum J.Sm. & Helecho epífito & BMP \\
\hline Elaeocarpaceae & Sloanea sp. & Árbol & BAP \\
\hline \multirow[t]{2}{*}{ Erythroxylaceae } & Erythroxylum citrifolium A.St.-Hil. & Árbol & BMP \\
\hline & Alchornea discolor Poepp. & Árbol & BMP \\
\hline \multirow[t]{10}{*}{ Euphorbiaceae } & Aparisthmium cordatum (A.Juss.) Baill. & Árbol & BAP \\
\hline & Conceveiba guianensis Aubl. & Árbol & BAP \\
\hline & Croton palanostigma Klotzsch & Árbol & BMP \\
\hline & Hyeronima oblonga (Tul.) Müll.Arg. & Árbol & BMP, BAP \\
\hline & Mabea aff. nitida Spruce ex Benth. & Árbol & BAP \\
\hline & Mabea subsessilis Pax. \& Hoffm. & Árbol & BAP \\
\hline & Maprounea guianensis Aubl. & Árbol & BAP \\
\hline & Micrandra rossiana R.E.Schult & Árbol & BMP, BAP \\
\hline & Plukenetia loretensis Ule & Trepadora & BAP \\
\hline & Sagotia brachysepala (Müll. Arg.) Secco & Árbol & BAP \\
\hline \multirow[t]{6}{*}{ Fabaceae } & Alexa confusa Pittier & Árbol & BMP, BAP \\
\hline & Alexa cowanii Yakovlev & Árbol & BMP \\
\hline & Andira surinamensis (Bondt.) Splitg. ex Amshoff & Árbol & BAP \\
\hline & Clathotropis aff. brachypetala (Tul.) Klein & Árbol & BAP \\
\hline & Diplotropis purpurea (Rich.) Amshoff & Árbol & BAP \\
\hline & Machaerium madeirense Pittier & Liana & BMP \\
\hline
\end{tabular}


Tabla 2 - Continuación

\begin{tabular}{|c|c|c|c|}
\hline Familia & Especie & Hábito & Ambiente \\
\hline & Swartzia piarensis R.S.Cowan & Árbol & BMP, BAP \\
\hline Flacourtiaceae & Casearia javitensis Kunth & Árbol & BMP \\
\hline \multirow[t]{3}{*}{ Gentianaceae } & Voyria acuminata Benth. & Hierba saprófita & BMP \\
\hline & Voyria aphylla (Jacq.) Pers. & Hierba saprófita & BMP, BAP \\
\hline & Voyriella parvifolia (Miq.) Miq. & Hierba saprófita & BMP \\
\hline Gesneriaceae & Codonanthe crassifolia (Focke) Morton & Trepadora epífita & BMP \\
\hline Gnetaceae & Gnetum leyboldii Tul. & Liana & BMP \\
\hline Grammitidaceae & Cochlidium furcatum (Hook. \& Grev.) C.Chr. & Helecho epífito & BAP \\
\hline Heliconiaceae & Heliconia acuminata Rich. & Hierba & BMP \\
\hline Icacinaceae & Discophora guianensis Miers & Árbol & BMP, BAP \\
\hline Ixonanthaceae & Ochthocosmus roraimae Benth. & Árbol & BMP \\
\hline \multirow[t]{10}{*}{ Lauraceae } & Aiouea myristicoides Mez & Árbol & BMP, BAP \\
\hline & Aniba parunensis (Meiss.) Mez & Árbol & BAP \\
\hline & Aniba taubertiana Mez & Árbol & BAP \\
\hline & Endlicheria aff. anomala (Nees) Mez & Árbol & BMP, BAP \\
\hline & Ocotea aff. cernua (Nees) Mez & Árbol & BMP \\
\hline & Ocotea aff. cujumari Mart. & Árbol & BMP \\
\hline & Ocotea neesiana (Miq.) Kosterm. & Árbol & BMP, \\
\hline & Ocotea percurrens Vicentini & Árbol & BMP \\
\hline & Ocotea schomburgkiana (Nees) Mez & Árbol & BMP \\
\hline & Ocotea splendens Mez & Árbol & BMP, BAP \\
\hline Lecythidaceae & Eschweilera coriacea (DC.) S.A.Mori & Árbol & BMP, BAP \\
\hline Loranthaceae & Oryctanthus alveolatus (Kunth) Kuijt & Parásita & BAP \\
\hline \multirow[t]{2}{*}{ Malpighiaceae } & Byrsonima concinna Benth. & Árbol pequeño & BBSAR \\
\hline & Hiraea fagifolia (DC.) A.Juss. & Liana & BAP \\
\hline \multirow[t]{4}{*}{ Marantaceae } & Calathea acuminata Steyerm. & Hierba & $\begin{array}{l}\text { BMP, lugares bajos y } \\
\text { húmedos }\end{array}$ \\
\hline & Ischnosiphon longiflorus K. Schum. & Hierba & $\begin{array}{l}\text { BMP, lugares bajos y } \\
\text { húmedos }\end{array}$ \\
\hline & Monotagma spicatum (Aubl.) Macbr. & Hierba & $\begin{array}{l}\text { BMP, lugares bajos y } \\
\text { húmedos }\end{array}$ \\
\hline & Monotagma tomentosum K. Schum. ex Loes. & Hierba & $\begin{array}{l}\text { BMP, lugares bajos y } \\
\text { húmedos }\end{array}$ \\
\hline Marattiaceae & Danaea simplicifolia Rudge & Helecho terrestre & BAP \\
\hline \multirow[t]{14}{*}{ Melastomataceae } & Clidemia involucrata DC. & Arbusto & BMP, sotobosque \\
\hline & Leandra divaricata (Naudin) Cogn. & Arbusto & BAP \\
\hline & Loreya mespiloides Miq. & Árbol & BAP, en borde \\
\hline & Maietia guianensis Aubl. & Arbusto & BAP \\
\hline & Maietia poeppiguii Mart. ex Cogn. & Arbusto & BAP \\
\hline & Miconia aff. chrysophyla (Rich.) Urb. & Árbol & BMP \\
\hline & Miconia ceramicarpa (DC.) Cogn. & Arbusto & BAP \\
\hline & Miconia holosericea (L.) DC. & Árbol & BMP \\
\hline & Miconia impetiolaris (Sw.) D.Don & Árbol & BAP \\
\hline & Miconia prasina (Sw.) DC. & Árbol & BAP \\
\hline & Miconia pubipetala Miq. & Árbol & BAP \\
\hline & Miconia tetraspermoides Wurdack & Árbol & BMP \\
\hline & Miconia tomentosa Rich.) D.Don ex DC. & Árbol & BAP \\
\hline & Mouriri brevipes Hook. & Árbol & BMP \\
\hline \multirow[t]{2}{*}{ Meliaceae } & Trichilia septentrionalis C.DC. & Árbol & BAP \\
\hline & Trichilia schomburgkii C.DC. & Árbol & BAP \\
\hline Menispermaceae & Abuta obovata Diels & Liana & BAP \\
\hline
\end{tabular}


Tabla 2 - Continuación

\begin{tabular}{|c|c|c|c|}
\hline Familia & Especie & Hábito & Ambiente \\
\hline Metaxiaceae & Metaxia rostrata (Kunth) L.Presl. & Helecho trepador & BMP \\
\hline \multirow[t]{5}{*}{ Mimosaceae } & Enterolobium schomburgkii Benth. & Árbol & BAP \\
\hline & Inga alba (Sw.) Willd. & Árbol & BAP \\
\hline & Inga lateriflora Miq. & Árbol & BMP, BAP \\
\hline & Inga multijuga Benth. & Árbol & BMP \\
\hline & Pentaclethra macroloba (Willd.) Kuntze & Árbol & BAP \\
\hline Monimiaceae & Siparuna reginae (Tul.) A.DC. & Árbol & BAP \\
\hline \multirow[t]{9}{*}{ Moraceae } & Brosimum alicastrum $\mathrm{Sw}$. & Árbol & BAP \\
\hline & Clarisia ilicifolia (Spreng.) Lanj. & Árbol & BAP \\
\hline & Ficus gomelleira Kunth \& Bouché & Árbol & BAP \\
\hline & Helicostylis scabra (J.F.Macbr.) C.C.Berg & Árbol & BMP \\
\hline & Clarisia ilicifolia (Spreng.) Lanj. & Árbol & BAP \\
\hline & Pseudolmedia laevigata Trécul. & Árbol & BAP \\
\hline & Pseudolmedia laevis (Ruiz \& Pav.) J.F.Macbr. & Árbol & BAP \\
\hline & Sorocea muriculata Miq. & Árbol & BAP \\
\hline & Trymatococcus amazonicus Poepp. \& Endl. & Árbol & BAP \\
\hline \multirow[t]{4}{*}{ Myristicaceae } & Iryanthera hostmannii (Benth.) Warb. & Árbol & BAP \\
\hline & Iryanthera juruensis Warb. & Árbol & BAP \\
\hline & Osteophloeum platyspermum (Spruce ex A.DC.) Warb. & Árbol & BAP \\
\hline & Virola elongata (Benth.) Warb. & Árbol & BAP \\
\hline \multirow[t]{4}{*}{ Myrtaceae } & Eugenia egensis DC. & Árbol & BAP \\
\hline & Myrcia bracteata (Rich.) DC. & Árbol & BAP \\
\hline & Myrcia paivae 0.Berg & Árbol & BMP \\
\hline & Myrcia pyrifolia (Desv. ex Ham.) Nied. & Árbol & BMP \\
\hline Olacaceae & Heisteria aff. ovata Benth. & Árbol & BMP \\
\hline Orchidaceae & Dichaea aff. trinitensis Gleason & Hierba epífita & BMP \\
\hline Piperaceae & Piper cililimbum Yunck. & Arbusto & BAP \\
\hline Poaceae & Olyra micrantha Kunth & Hierba & BMP, BAP sotobosque \\
\hline \multirow[t]{2}{*}{ Polygalaceae } & Bredemeyera sp. & Liana & BMP \\
\hline & Moutabea guianensis Aubl. & Liana & BMP \\
\hline \multirow{2}{*}{ Polypodiaceae } & Microgramma aff. lycopodioides (L.) Copel. & Helecho trepador & BAP \\
\hline & Polypodium aff. triseriale Sw. & Helecho terrestre & BAP \\
\hline \multirow[t]{2}{*}{ Quiinaceae } & Quiina guianensis Aubl. & Árbol & BAP \\
\hline & Quiina obovata Tul. & Árbol & BAP \\
\hline \multirow[t]{2}{*}{ Rizophoraceae } & Cassipourea guianensis Aubl. & Árbol & BMP \\
\hline & Sterigmapetalum guianense Steyerm. & Árbol & BMP \\
\hline \multirow[t]{13}{*}{ Rubiaceae } & Faramea sp. & Arbusto & BAP \\
\hline & Palicourea nitidella (Müll.Arg.) Standl. & Arbusto & BMP \\
\hline & Psychotria deflexa DC. & Arbusto & BMP \\
\hline & Psychotria hoffmannseggiana (Willd. ex Roem \& Schult) Müll.Arg. & Arbusto & BMP \\
\hline & Psychotria iodotricha Müll. Arg. & Arbusto & BMP \\
\hline & Psychotria phaneroloma Standl. \& Steyerm. & Arbusto & BMP \\
\hline & Psychotria platypoda A.DC. & Arbusto & BAP \\
\hline & Psychotria poeppigiana Müll.Arg. & Arbusto & BMP \\
\hline & Psychotria poliycephala Benth. & Arbusto & BAP \\
\hline & Psychotria racemosa (Aubl.) Raeusch. & Arbusto & BAP \\
\hline & Psychotria ulviformis Steyerm. & Hierba rastrera & BAP \\
\hline & Psychotria variegata Steyerm. & Hierba rastrera & BMP, BAP \\
\hline & Remijia roraimae (Benth.) K.Schum. & Árbol & BMP \\
\hline \multirow[t]{3}{*}{ Sapindaceae } & Talisia chartacea Acev.-Rodr. & Árbol & BMP \\
\hline & Talisia macrophylla Radlk. & Árbol & BAP \\
\hline & Toulicia sp. & Árbol & BAP \\
\hline
\end{tabular}


Tabla 2 - Continuación

\begin{tabular}{llll}
\hline Familia & Especie & Hábito & Ambiente \\
\hline Sapotaceae & Micropholis guianensis (A.DC.) Pierre & Árbol & BAP \\
& Pouteria eugenifolia (Pierre) Bahehni & Árbol & BMP \\
\hline Schizaeseceae & Pouteria guianensis & Árbol & BAP \\
\hline Solanaceae & Schizaea elegans (Vahl) Sw. & Helecho terrestre & BMP \\
Violaceae & Markea reticulata Steyerm. & Liana epífita & BAP \\
& Amphirrhox longifolia (St.Hil.) Spreng. & Árbol & BAP \\
& Leonia cymosa Mart. & Árbol & BMP \\
\hline Viscaceae & Rinorea flavescens (Aubl.) O.Kuntze & Árbol & BMP \\
Vochysiaceae & Rinorea macrocarpa (Mart. ex Eichler) Kuntze & Árbol & BAP \\
& Phoradendron crassifolium (DC.) Eichl. & Parásita & BAP \\
\hline & Erisma uncinatum Warm. & Árbol & BMP, BAP \\
\hline
\end{tabular}

BMP: Bosque medio en pendiente; BAP: Bosque alto en penillanura; BM: Bosque medio

En el área estudiada los bosques altos se encuentran en peniplanicies de topografía plana a ligeramente ondulada. Predominan árboles entre 25 y $30 \mathrm{~m}$, con troncos rectos, con ramificaciones principales medias y altas, y algunas raíces tabulares. El estrato superior forma una estructura discontinua, donde las copas no se tocan, con elementos emergentes de hasta $38 \mathrm{~m}$. Los estratos medios y bajos por el contrario, son continuos. Las especies arbóreas más abundantes son Eperua jenmanii, Micrandra rossiana, Alexa confusa, Rinorea flavescens, Crepidospermum rhoifolium y Amphirrhox longifolia. En algunos microambientes más húmedos del bosque se pueden observar epífitas como Philodendron fragantissimum, Anthurium clavigerum y A. gracile. El sotobosque tiene con una capa de hojarasca de aproximadamente $10 \mathrm{~cm}$ de grosor. Está formado por plantas herbáceas y arbustivas como Heliconia psitacorum, Piper demeraranum, P. piscatorum, Maieta guianensis, M. poeppigii y Miconia ceramicarpa; en algunos sectores más abiertos del sotobosque se pueden encontrar agrupaciones de Psychotria variegata y P. ulviformis.

Los bosques medios del área se localizan preferentemente en planos inclinados de hasta $45 \%$. En las comunidades estudiadas los tallos son en su mayoría rectos y delgados y la densidad es mayor en los sectores de mayor pendiente. En los menos inclinados se observan troncos más espaciados y altos. La altura de los árboles varía entre 20 y $25 \mathrm{~m}$. Aunque el dosel es abierto, los estratos inferiores y medios son más cerrados que en los bosques altos. Las especies arbóreas más abundantes son Ochthocosmus roraimae, Conceveiba guianensis, Bocageopsis multiflora, Swartzia piarensis, Protium trifoliolatum, Caraipa tereticaulis y Tachigali guianensis y las lianas Machaerium madeirense, Arrabidaea nigrescens y Heteropsis flexuosa. El sotobosque es muy denso, con una capa gruesa de hojarasca y detritos vegetales de hasta $20 \mathrm{~cm}$. Predominan especies arbustivas como Aphelandra pulcherrima, varias especies del género Psychotria, y hierbas como Olyra micrantha, Heliconia acuminata, Calathea acuminata, Ischnosiphion longiflorus y Diplasia karataefolia, así como la palma Bactris simplicifrons.

En ambos bosques en época de lluvias, es común observar en la hojarasca especies saprófitas como Gymnosiphon cymosus, G. divaricatus, Voyria acuminata y V. amphylla.

\section{MORICHAL}

En el morichal estudiado el estrato arbóreo de la vegetación está compuesto por una sola especie, la palma moriche Mauritia flexuosa, que alcanza unos $12 \mathrm{~m}$ de alto, y un estrato bajo de hasta $2 \mathrm{~m}$ de especies arbustivas y herbáceas anegadas por la corriente de un riachuelo de aguas claras. Se inventariaron 18 familias, 31 géneros y 33 especies de plantas vasculares, entre las que destaca la familia Cyperaceae con 7 especies (Tabla 3). Se observan arbustos como Rhynchanthera grandifolia, Turnera acuta, hierbas como Chelonanthus alatus, Urospatha sagitiifolia, Pterogastra divaricata, Buchnera palustris, Rynchospora unisetosa, Rhytachne rottboellioides, Eriocaulon humboldtii, Websteria submersa, Conobea acuatica y trepadoras como Malanea obovata, Securidaca marginata y Mandevilla hirsuta. En el ecotono morichal-sabana se pueden encontrar hierbas y sufrútices como Echinolaena inflexa, Hyptis atrorubens, Sipanea pratensis, Melananthus ulei, Polygala spruceana y Macairea lasiophylla.

\section{SABANA}

Las sabanas están rodeadas por elementos de bosques y en muchos casos cercanas a los cursos de los ríos. En este ambiente se inventariaron 17 familias, 31 géneros y 35 especies de plantas vasculares, entre las que se abundan especies de Poaceae (8) y de Cyperaceae (7) (Tabla 4). Destacan especies leñosas como Byrsonima crassifolia, Roupala montana y Ternstroemia pungens. Como elementos herbáceos abundantes 


\begin{tabular}{|c|c|c|c|}
\hline Familia & Especie & Hábito & Ambiente \\
\hline Apocynaceae & Mandevilla hirsuta (Rich.) K.Schum. & Trepadora & Lugar anegado \\
\hline Arecaceae & Mauritia flexuosa L.f. & Palma arborescente & Lugar anegado \\
\hline \multirow[t]{7}{*}{ Cyperaceae } & Eleocharis pachytylla (C.Wright) C.B.Clark & Hierba & Ecotono sabana-Morichal \\
\hline & Hypolytrum pulchrum (Rudge) H. Pfeiff. & Hierba & Lugar anegado \\
\hline & Lagenocarpus rigidus (Kunth) Nees & Hierba & Lugar anegado \\
\hline & Rynchospora unisetosa T.Koyama & Hierba & Lugar anegado \\
\hline & Rynchospora filiformis Vahl & Hierba & Lugar anegado \\
\hline & Rynchospora globosa (Kunth) Roem. \& Schult. & Hierba & Lugar anegado \\
\hline & Websteria submersa (C.Wright) Britton & Hierba & Lugar anegado \\
\hline Araceae & Urospatha sagittifolia (Rudge) Schott & Hierba & Lugar anegado \\
\hline Clusiaceae & Mahurea exstipulata Benth. & Árbol & Lugar anegado \\
\hline Eriocaulaceae & Eriocaulon humboldtii Kunth & Hierba & Lugar anegado \\
\hline \multirow[t]{2}{*}{ Gentianaceae } & Chelonanthus alatus (Aubl.) Pulle & Sufrútice & Lugar anegado \\
\hline & Coutoubea reflexa Benth. & Hierba & Lugar anegado \\
\hline Lamiaceae & Hyptis atrorubens Poit. & Hierba & Lugar anegado \\
\hline Lentibulariaceae & Genlisea guianensis N.E.Br. & Hierba & Lugar anegado \\
\hline \multirow[t]{3}{*}{ Melastomataceae } & Desmoscelis villosa (Aubl.) Naudin & Sufrútice & Lugar anegado \\
\hline & Rynchantera grandiflora (Aubl.) DC. & Arbusto & Lugar anegado \\
\hline & Pterogastra divaricada (Bonpl.) Naudin & Hierba & Lugar anegado \\
\hline Ochnaceae & Sauvagesia rubiginosa St.Hil. & Sufrútice & Lugar anegado \\
\hline Onagraceae & Ludwigia nervosa (Poir.) H.Hara & Sufrútice & Lugar anegado \\
\hline Orchidaceae & Otostylis lepida (Linden \& Rchb.f) Schltr. & Hierba & Borde de morichal \\
\hline \multirow[t]{3}{*}{ Poaceae } & Echinolaena inflexa (Poir.) Chase & Hierba & Borde de morichal \\
\hline & Ischaemum guianense Kunth & Hierba & Lugar anegado \\
\hline & Rhytachne rottboellioides Desv. & Hierba & Lugar anegado \\
\hline \multirow[t]{2}{*}{ Polygalaceae } & Polygala spruceana A.W.Benn. & Hierba & $\begin{array}{l}\text { Ecotono húmedo sabana- } \\
\text { morichal }\end{array}$ \\
\hline & Securidaca marginata Benth. & Liana & Borde de Morichal \\
\hline \multirow[t]{4}{*}{ Rubiaceae } & Perama galioides (Kunth) Poir. & Hierba & $\begin{array}{l}\text { Ecotono húmedo sabana- } \\
\text { morichal }\end{array}$ \\
\hline & Sipanea pratensis Aubl. & Sufrútice & $\begin{array}{l}\text { Ecotono húmedo sabana- } \\
\text { morichal }\end{array}$ \\
\hline & Malanea obovata Hochr. & Liana & Lugar anegado \\
\hline & Sipanea pratensis Aubl. & Sufrútice & $\begin{array}{l}\text { Ecotono húmedo sabana- } \\
\text { morichal }\end{array}$ \\
\hline \multirow[t]{2}{*}{ Schrophulariaceae } & Conobea acuatica Aubl. & Hierba & Lugar anegado \\
\hline & Buchnera palustris (Aub.) Spreng. & Hierba & Lugar anegado \\
\hline Sterculiaceae & Byttneria scabra L. & Sufrutice & Lugar anegado \\
\hline
\end{tabular}

se encontraron Paspalum lanciflorum, Hypolytrum pulchrum y Echinolaena inflexa. En los límites de la sabana, muy cerca de ríos, el sustrato suele ser muy húmedo y frecuentemente anegado en época de lluvia, lo cual favorece la formación de agrupaciones de Utricularia cf. pusilla, Scleria verticillata, Rynchospora unisetosa, así como individuos de Polygala adenophora y Buchnera palustris. En lugares más elevados y secos se puede encontrar Schiekia orinocensis y Palicourea rigida.

\section{ARBUSTAL}

Este tipo de vegetación se encuentra sobre sustrato arenoso y está formada por elementos leñosos de bajo porte, no mayor de $5 \mathrm{~m}$ de alto, cuyos troncos presentan varias ramificaciones, y por algunos elementos herbáceos que se disponen de manera dispersa. Se inventariaron 26 familias, 46 géneros y 55 especies (Tabla 5), destacando las Rubiaceae (8 especies), Melastomataceae (5), y Myrtaceae y Orchidaceae (4). Como elementos arbustivos importantes se pueden señalar las especies 


\begin{tabular}{|c|c|c|c|}
\hline Familia & Especie & Hábito & Ambiente \\
\hline \multirow[t]{2}{*}{ Caesalpiniaceae } & Chamaechrista flexuosa (L.) Greene & Súfrutice & Sabana intervenida por quema \\
\hline & Chamaechrista diphylla (L.) Greene & Sufrútice & Sabana \\
\hline \multirow[t]{7}{*}{ Cyperaceae } & Bulbostylis juncoides (Vahl) Kük ex Osten & Hierba & En borde húmedo sabana-morichal \\
\hline & Eleocharis pachystila (C. Wright) C.B Clark & Hierba & Lugares húmedos \\
\hline & Hypolytrum pulchrum (Rudge) H.Pfeiff. & Hierba & Sabana \\
\hline & Lagenocarpus rigidus (Kunth) Nees & Hierba & Sabana \\
\hline & Rhynchospora globosa (Kunth) Roem. \& Schult. & Hierba & Sabana \\
\hline & Rhynchospora unisetosa T. Koyama & Hierba & Sabana \\
\hline & Scleria verticilata Muhl ex Willd. & Hierba & Lugar anegado \\
\hline \multirow[t]{2}{*}{ Gentianaceae } & Coutoubea ramosa Aubl. & Hierba & Lugares húmedos \\
\hline & Irlbachia nemorosa (Willd. ex Roem. \& Schult.) Merrill & Hierba & En lugares muy húmedos \\
\hline Haemodoraceae & Schiekia orinocensis (.Kunth) Meisn. & Hierba & Sabana \\
\hline Lamiaceae & Hyptis atrorubens Poit. & Hierba & Lugares húmedos \\
\hline Lycopodiaceae & Lycopodiella cernua (L.) Pic.Serm. & Hierba & Borde húmedo sabana morichal \\
\hline \multirow[t]{2}{*}{ Malpighiceae } & Byrsonima crassifolia (L.) Kunth & Árbol pequeño & Sabana \\
\hline & Byrsonima verbascifolia (L.) Rich. & Arbusto & Sabana \\
\hline Melastomataceae & Miconia alborufescens (Aubl.) DC. & Arbusto & Sabana \\
\hline Passifloraceae & Passiflora auriculata Kunth & Trepadora & En el borde bosque-sabana \\
\hline \multirow[t]{8}{*}{ Poaceae } & Axonopus aureus Beauv. & Hierba & Sabana \\
\hline & Axonopus anceps (Mez) Hitchc. & Hierba & Sabana \\
\hline & Echinolaena inflexa (Poir.) Chase & Hierba & Sabana \\
\hline & Leptocoryphium lanatum (Kunth) Nees & Hierba & Sabana \\
\hline & Panicun cyanescens Nees in Trin & Hierba & Sabana \\
\hline & Paspalum lanciflorum Trin & Hierba & Sabana, abundante \\
\hline & Trachypogon plumosus (Humb. \& Bonpl.) Nees & Hierba & Sabana \\
\hline & Rhytachne rottboellioides Desv. & Hierba & Sabana \\
\hline Polygalaceae & Polygala spruceana A.W. Benn. & Hierba & Lugares húmedos \\
\hline Proteaceae & Roupala montana Aubl. & Árbol pequeño & En el borde de la sabana \\
\hline \multirow[t]{3}{*}{ Rubiaceae } & Palicourea rigida Kunth & Sufrútice & En lugar seco de la sabana \\
\hline & Perama galioides (Kunth) Poir. & Hierba & Lugares húmedos \\
\hline & Sipanea pratensis Aubl. & Sufrútice & Lugares húmedos \\
\hline Solanaceae & Melananthus ulei Carvalho & Sufrútice & Lugares húmedos \\
\hline Sterculiaceae & Byttneria scabra L. & Sufrútice & Sabana \\
\hline Schrophulariaceae & Buchnera palustris (Aubl.) Spreng. & Hierba & Lugares anegados \\
\hline
\end{tabular}

Pradosia schomburgkiana, Humiria balsamifera, Schitostemon auyantepuyensis, Emmotum conjunctum, Byrsonima concinna y Graffenrieda sessilifolia. Entre los elementos herbáceos y sufruticosos se encuentran Borreria verticilata, Irlbachia cardonae, Phyllanthus vaccinifolius, Sobralia liliastrum, Philodendron callosus y Lagenocarpus glomeratus. Cabe destacar la presencia de agregaciones de Siphanthera hostmanni y Perama dichotoma en lugares sombreados y húmedos.

\section{AMBIENTES PERTURBADOS}

En la parte baja de la cuenca del Río Cucurital se pueden encontrar ambientes intervenidos, ya sea por la elaboración de conucos o por construcciones de interés turístico. Se encontraron 20 familias, 33 géneros y 33 especies, destacando las familias Melastomataceae (10 especies) y Rubiaceae (4), así como especies del género Clidemia (5) (Tabla 6). Estos lugares están colonizados por especies herbáceas, sufruticosas y arbustivas, entre las que se pueden señalar arbustos como Siparuna guianensis, Tapirira guianensis, Vismia guianensis, Clidemia urceolata y C. sericea, así como hierbas como Zornia latifolia, Polygala timotou, Microstachys corniculata, Siphanthera hostmannii, Hyptis lantanifolia, Rynchospora cephalotes y Comolia microphylla. 
Tabla 5 - Especies presentes arbustal de la cuenca baja del Río Cucurital

\begin{tabular}{|c|c|c|c|}
\hline Familia & Especie & Hábito & Ambiente \\
\hline \multirow[t]{3}{*}{ Araceae } & Anthurium guayanum G.S.Bunting & Hierba & En sustrato rocoso \\
\hline & Philodendron deflexum Poepp. ex Schott & Hierba & Sustrato arenoso en pendiente \\
\hline & Philodendron callosum K.Krause & Hierba & Sobre rocas \\
\hline \multirow[t]{2}{*}{ Asteraceae } & Lepidaploa ehrectifolia (Benth.) H.Rob. & Arbusto & Sustrato arenoso \\
\hline & Pectis sp. & Arbusto & Sustrato arenoso en pendiente \\
\hline Bignoniaceae & Distictella pauciflora A.H.Gentry & Liana & Sustrato arenoso en pendiente \\
\hline \multirow[t]{3}{*}{ Clusiaceae } & Clusia schomburgkiana (Planch. \& Triana) Benth. ex Engl. & Árbol & Parte baja anegada \\
\hline & Clusia sp. (sección Oedematopus) & Árbol & Sustrato arenoso en pendiente \\
\hline & Mahurea exstipulata Benth. & Árbol & Parte baja anegada \\
\hline \multirow{2}{*}{ Cyperaceae } & Lagenocarpus glomeratus Gilly & Hierba & Sustrato arenoso en pendiente \\
\hline & Rynchospora rugosa (Vahl) Gale & Hierba & Parte baja anegada \\
\hline Dilleniaceae & Davilla kunthii A.St.Hill. & Trepadora & Parte baja anegada \\
\hline Dryopteridaceae & Elaphoglossum plumosum (Fée) T.Moore & Helecho epífito & Sustrato arenoso y rocoso \\
\hline \multirow[t]{2}{*}{ Ericaceae } & Styria parunensis (Benth.) Benth. \& Hook. & Arbusto & Sustrato arenoso \\
\hline & Vaccinium euryanthum A.C.Sm. & Arbusto & Sustrato arenoso \\
\hline \multirow[t]{2}{*}{ Eriocaulaceae } & Paepalanthus fasciculatus (Rottb.) Kunth & Hierba & Sobre rocas, en lugar muy húmedo \\
\hline & Singonanthus longipes Gleason & Hierba & Parte baja anegada \\
\hline Euphorbiaceae & Phyllanthus vacciniifolius (Müll.Arg.) Müll.Arg. & Sufrútice & Sustrato arenoso \\
\hline Euphroniaceae & Euphronia guianensis (R.H.Schomb.) Hallier & Árbol & Sustrato arenoso \\
\hline Fabaceae & Taralea crassifolia Benth. & Árbol & Sustrato arenoso \\
\hline \multirow[t]{2}{*}{ Gentianaceae } & Irlbachia cardonae (Gleason) Maguire & Hierba & Sustrato arenoso \\
\hline & Irlbachia nemorosa (Willd. ex Roem. \& Schult.) Merrill & Hierba & Sustrato arenoso \\
\hline \multirow[t]{3}{*}{ Humiriaceae } & Humiria balsamifera (Aubl.) St. Hil. & Arbusto & Sustrato arenoso \\
\hline & Sacoglottis guianensis Benth. & Árbol & Sustrato arenoso \\
\hline & Schistostemon auyentepuiensis Cuatrec. & Arbusto & Sustrato arenoso \\
\hline Icacinaceae & Emmotum conjunctum R.A. Howard & Arbusto & Sustrato arenoso \\
\hline \multirow[t]{2}{*}{ Malpighiaceae } & Byrsonima concinna Benth. & Árbol pequeño & Sustrato arenoso \\
\hline & Pterandra sericea W.R.Anderson & Árbol pequeño & Sustrato arenoso y rocoso \\
\hline Marcgraviaceae & Sarcopera flammifera de Roon \& Bedell & Trepadora & Sustrato arenoso \\
\hline \multirow[t]{5}{*}{ Melastomataceae } & Aciotis indecora (Bonpl.) Triana & Sufrútice & Sustrato arenoso \\
\hline & Graffenrieda sessilifolia Triana & Arbusto & Sustrato arenoso \\
\hline & Macairea pachyphylla Benth. & Árbol & Sustrato arenoso \\
\hline & Rhynchanthera grandiflora (Aubl) DC. & Arbusto & Parte baja anegada \\
\hline & Siphanthera hostmannii Cogn. & Hierba & Formando colonia sobre roca \\
\hline Mimosaceae & Calliandra surinamensis Ben th. & Arbusto & Sustrato arenoso y rocoso \\
\hline Myrsinaceae & Cybianthus spicatus (Kunth) G.Agostini & Arbusto & Sustrato arenoso \\
\hline \multirow[t]{4}{*}{ Myrtaceae } & Marlierea karuaiensis (Steyerm.) McVaugh & Arbusto & Sustrato arenoso \\
\hline & Myrcia citrifolia (Aubl.) Urb. & Árbol & Sustrato rocoso \\
\hline & Myrcia fallax (Rich.) DC. & Árbol & Sustrato arenoso \\
\hline & Myrcia sylvatica (G.Mey.) DC. & Arbusto & Sustrato arenoso \\
\hline \multirow[t]{4}{*}{ Orchidaceae } & Brifenaria Iongicornis Lindl. & Hierba epífita & Sustrato rocoso \\
\hline & Epistephium subrepens Hoehne & Hierba & Parte baja, inundada de arbustal \\
\hline & Phragmipedium klotzscheanum (Rchb.f.) Rolfe & Hierba & Sustrato arenoso y rocoso \\
\hline & Sobralia liliastrum Lindl. & Hierba & Sustrato arenoso \\
\hline \multirow[t]{6}{*}{ Rubiaceae } & Borreria verticilata (L.) Mey. & Sufrutice & Sustrato arenoso \\
\hline & Chiococca nitida Benth. & Arbusto & Sustrato arenoso y rocoso \\
\hline & Coccocypselum guianense (Aubl.) Schum. & Hierba rastrera & $\begin{array}{l}\text { Suatrato arenoso, húmedo y } \\
\text { sombreado }\end{array}$ \\
\hline & Geophyla orbicularis (Müll.Arg.) Steyerm. & Hierba rastrera & En lugar sombreado \\
\hline & Perama dichotoma Poepp. \& Endl. & Hierba & Sobre rocas \\
\hline & Perama galioides (Kunth) Poir. & Hierba & Lugares arenosos húmedos \\
\hline
\end{tabular}


Tabla 5 - Continuación

\begin{tabular}{llll}
\hline Familia & Especie & Hábito & Ambiente \\
\hline & Retiniphyllum concolor (Spruce ex Benth.) Müll.Arg. & Arbusto & Sustrato arenoso \\
& Retiniphyllum schomburgkii (Benth.) Müll.Arg. & Arbusto & Sustrato arenoso \\
Sapotaceae & Pradosia schomburgkiana (A.DC.) Cronquist & Árbol & Sustrato arenoso en pendiente \\
Vochysiaceae & Ruizterania ferruginea (Steyerm.) Marc.-Berti & Arbol & Sustrato arenoso \\
Xyridaceae & Xyris fallax Malme & Hierba & Parte baja anegada \\
\hline
\end{tabular}

Tabla 6 - Especies que se pueden encontrar colonizando ambientes perturbados

\begin{tabular}{|c|c|c|c|}
\hline Familia & Especie & Hábito & Ambiente \\
\hline Anacardiaceae & Tapirira guianensis Aubl. & Árbol pequeño & Intervenido por actividad turística \\
\hline Annonaceae & Xylopia aromatica (Lam.) Mart. & Árbol & Intervenido por actividad turística \\
\hline Asteraceae & Conyza bonariensis (L.) Cronquist & Hierba & En conuco abandonado \\
\hline Caesalpiniaceae & Chamaechrista flexuosa (L.) Greene & Sufrútice & Intervenido por actividad turística \\
\hline Clusiaceae & Vismia japurensis Reichard in Mart. & Arbol pequeño & En conuco abandonado \\
\hline Clusiaceae & Vismia guianensis (Aubl.) Choisy & Arbusto & En conuco abandonado \\
\hline Cyperaceae & Cyperus laxus Lam. & Hierba & Intervenido por actividad turística \\
\hline Cyperaceae & Rhynchospora cephalotes (L.) Vahl & Hierba & Intervenido por actividad turística \\
\hline Euphorbiaceae & Maprounea guianensis Aubl. & Árbol & Intervenido por actividad turística \\
\hline Euphorbiaceae & Mycrostachys corniculata (Vahl) Griseb. & Sufrútice & Intervenido por actividad turística \\
\hline Fabaceae & Abrus precatorius $\mathrm{L}$. & Trepadora & Intervenido por actividad turística \\
\hline Fabaceae & Desmodium ascendens (Sw.) DC. & Súfrutice & Intervenido por actividad turística \\
\hline Fabaceae & Zornia latifolia Sm. in Rees & Hierba & Intervenido por actividad turística \\
\hline \multirow[t]{2}{*}{ Gentianaceae } & Chelonanthus angustifolius (H.B.K.) Gilg & Hierba & En conuco abandonado \\
\hline & Coutoubea ramosa Aubl. & Hierba & En conuco abandonado \\
\hline Heliconiaceae & Heliconia psitacorum L.f. & Hierba & Intervenido por actividad turística \\
\hline Lamiaceae & Hyptis lantanifolia Poit. & Hierba & Intervenido por actividad turística \\
\hline \multirow[t]{10}{*}{ Melastomataceae } & Clidemia bullosa DC. & Arbusto & Intervenido por actividad turística \\
\hline & Clidemia capitellata (Bonpl.) D.Don & Arbusto & Conuco abandonado \\
\hline & Clidemia sericea D.Don & Hierba & Intervenido por actividad turística \\
\hline & Clidemia strigillosa (Sw.) DC. & Arbusto & Intervenido por actividad turística \\
\hline & Clidemia urceolata DC. & Arbusto & Intervenido por actividad turística \\
\hline & Comolia microphylla Benth. & Hierba & Intervenido por actividad turística \\
\hline & Macairea lasiophylla (Benth.) Wurdack & Hierba & En conuco abandonado \\
\hline & Miconia lepidota DC. & Árbol pequeño & Conuco abandonado \\
\hline & Miconia rufescens (Aubl.) DC. & Arbusto & Intervenido por actividad turística \\
\hline & Siphanthera hostmanii Cogn. & Hierba & Intervenido por actividad turística \\
\hline Monimiaceae & Siparuna guianensis Aubl. & Arbusto & Intervenido por actividad turística \\
\hline Poaceae & Schizachyrium sanguineum (Retz.) Alston & Hierba & Intervenido por actividad turística \\
\hline Polygalaceae & Polygala timotou Aubl. & Hierba & Intervenido por actividad turística \\
\hline Rhamnaceae & Gouania blanchetiana Mart. & Trepadora & Conuco abandonado \\
\hline \multirow[t]{4}{*}{ Rubiaceae } & Borreria latifolia (Aubl.) Schum. & Sufrútice rastrero & Conuco abandonado \\
\hline & Isertia hypoleuca Benth. & Arbol & Conuco abandonado \\
\hline & Psychotria poeppigiana (Müll. Arg.) Steyerm. & Arbusto & Conuco \\
\hline & Sipanea pratensis Aubl. & Sufrútice & En conuco abandonado \\
\hline \multirow[t]{2}{*}{ Solanaceae } & Solanum asperum Rich. & Arbusto & Conuco abandonado \\
\hline & Solanum subinerme Jacq. & Arbusto & $\begin{array}{l}\text { Vegetación secundaria en conuco } \\
\text { abandonado }\end{array}$ \\
\hline Viscaceae & Phoradendron crassifolium (DC.) Eichl. & Parásita & Intervenido por actividad turística \\
\hline
\end{tabular}




\section{ASPECTOS FLORÍSTICOS GENERALES}

En la cuenca baja del río Cucurital se identificaron 106 familias, 310 géneros y 520 especies de plantas vasculares, detallándose el resultado para cada grupo vegetal (Pteridófitas, Gimnospermas y Angiospermas) en la Tabla 7. Entre las familias de angiospermas destacan las Melastomataceae (46 especies), Rubiaceae (33), Euphorbiaceae (23), Cyperaceae (19), Myrtaceae (18), Fabaceae (18), Clusiaceae (15), Arecaceae (15), Caesalpiniaceae (14), Chrysobalanaceae (14), Lauraceae (14), Poaceae (13) y Burseraceae (12), siendo las más representativas de la cuenca.

Los géneros con mayor cantidad de especies son Miconia (17), Psychotria (13), Ocotea (10), Myrcia (8), Protium (7), Licania (7), y Rhynchospora (7). La predominancia de las Melastomataceae y Rubiaceae es debida principalmente a la abundancia de especies de porte herbáceo y arbustivo, las cuales están presentes en el sotobosque, sabanas, bosques ribereños y en la transición bosque-sabana. Cabe mencionar que estas familias están dentro de las que presentan mayor número de especies en la Guayana venezolana (Berry et al., 1995b), particularmente la abundancia de especies de estas familias ha sido señalada para un arbustal en Gran Sabana (Ramírez et al., 1988).

Tabla 7 - Número de familias, géneros y especies colectadas en la cuenca baja del Río Cucurital

\begin{tabular}{llll}
\hline Grupo vegetal & Familias & Géneros & Especies \\
\hline Helechos & 11 & 17 & 26 \\
Gimnospermas & 1 & 1 & 1 \\
Angiospermas & 94 & 292 & 493 \\
Dicotiledóneas & 77 & 231 & 396 \\
Monocotiledóneas & 17 & 61 & 97 \\
TOTAL & 106 & 310 & 520 \\
\hline
\end{tabular}

Tabla 8 - Especies de distribución restringida a la Guayana venezolana de acuerdo a (Steyemark et al.1995-2005) y encontradas en la cuenca baja del Río Cucurital.

\begin{tabular}{lll}
\hline Familia & Especie & Distribución \\
\hline Acanthaceae & Justicia kunthartii Leonard & Bolívar (Auyan-tepui, Canaima Guaiquinima) \\
\hline Apocynaceae & Spongiosperma cataractarum Zaruchi & Bolívar (Canaima, alto Río Paragua), Amazonas (Río Sipapo) \\
Araceae & Anthurium guayanum G.S.Bunting & Bolívar (Auyán-tepui, Canaima, Macizo del Chimantá, Sierra Ichún, Urimán). \\
\hline Araceae & Philodendron dunstervilleoum G.S.Bunting & Bolívar (Río Nichare, Salto Pará). \\
\hline Bignoniaceae & Distictella pauciflora A.H.Gentry & Bolívar (cerca de Canaima) \\
\hline Bombacaceae & Pachira gracilis (Robyns) W.S. Anderson & Bolívar (Auyán-tepui, Cerro Guaiquinima, Cerro Majarano, Cerro Sarisariñama) \\
\hline Caesalpiniaceae & Eperua venosa R.S.Cowan & Bolívar (Río Chicanán, Salto Ángel) \\
Chrysobalanaceae & Couepia foveolata Prance & Bolívar (alto Río Caroní, alto Río Paragua) \\
\hline Fabaceae & Swartzia piarensis R.S.Cowan & Bolívar (Cerro Bolívar, Río Caroní) \\
& Swartzia steyermarkii R.S. Cowan & Bolívar (Río Ayaiche, Río Uroi). \\
\hline Humiriaceae & Schistostemon auyantepuiensis Cuatrec. & Bolívar (Auyán-tepui, cerca de Guayaraca). \\
Malvaceae & Peltaea surumuensis (Ulbr.) Krapov. \& Cristóbal & Bolívar (Canaima, Gran Sabana, Río Aprada) \\
\hline
\end{tabular}


Tabla 8 - Continuación

\begin{tabular}{|c|c|c|}
\hline Familia & Especie & Distribución \\
\hline Melastomataceae & $\begin{array}{l}\text { Graffenrieda sessilifolia Triana subsp. cardonae } \\
\text { Wurdack }\end{array}$ & Bolívar (Auyán-tepui) \\
\hline Myrtaceae & Eugenia pubescens (Kunth) DC. & $\begin{array}{l}\text { Bolívar (cerca de Ciudad Bolívar, Río Caroní, Río Caura, Río Nichare, Río } \\
\text { Paragua), Amazonas (Medio Río Orinoco) }\end{array}$ \\
\hline \multirow[t]{2}{*}{ Piperaceae } & Piper dunstervilleorum Steyerm. & Bolívar (Río Cuyuní) \\
\hline & Piper piscatorum Trel. \& Yunck. & $\begin{array}{l}\text { Bolívar (medio Río Caura, Cerro Cuchivero, Río Cuyuní, Gran Sabana, El } \\
\text { Manteco, Río Guayapo, Río Parguaza), Amazonas (cerca de Río Parucito) }\end{array}$ \\
\hline Sapindaceae & Talisia chartacea Acev.-Rodr. & Bolívar Cerro Guaiquinima), Amazonas (entre Atabapo y La Esmeralda) \\
\hline Smilacaceae & Smilax pittieriana Steyerm. & $\begin{array}{l}\text { Bolívar (Altiplanicie de Nuria, Amaruay-tepui, Apacará-tepui, Cerro Guaiquinima, } \\
\text { Cerro Pitón, El Paují, Gran Sabana, Guayaraca, Icabarú, Río Oris, Río Tirica, } \\
\text { Sierra Auraima, Uaipán-tepui), Amazonas (Cerro Aracamuni, Cerro Yutajé, San } \\
\text { Carlos de Río Negro, Sierra Parima) }\end{array}$ \\
\hline Viscaceae & Dendrophthora lacryma-jobi E.A.Kellogg & $\begin{array}{l}\text { Bolívar (Auyán-tepui, Cerro Guaiquinima, Macizo del Chimantá, Río Tonoro, } \\
\text { Salto Uraima), Amazonas (Caño Caname, Cerro Marahuaka, Cerro Yutajé) }\end{array}$ \\
\hline
\end{tabular}

\section{CONCLUSIONES}

En la cuenca baja del Río Cucurital se encuentra una diversidad de ambientes con elementos florísticos característicos. Estos paisajes son representativos del Escudo Guayanés, principalmente en la región sureste del estado Bolívar. El asentamiento de comunidades indígenas en la cuenca baja del río Cucurital es muy bajo, lo cual repercute en la escasa intervención de las comunidades. Los pocos ambientes que han sido intervenidos, ya sea por interés turístico o por la realización de conucos de subsistencia, han sido colonizados por especies herbáceas y arbustivas típicas de la región. Sin embargo la cercanía a Canaima, zona de alto interés turístico y mayor presión poblacional, podría en un futuro alterar el carácter prístino de estos ambientes. Recientemente (año 2007), se desataron incendios masivos en el sector al sur de la cuenca del río Cucurital que afectaron cientos de hectáreas de vegetación herbácea y boscosa, inclusive tepuyana. Por tal razón, es recomendable considerar las medidas necesarias para prevenir la ocurrencia de tales eventos catastróficos y mitigar sus efectos a largo plazo.

\section{AGRADECIMIENTO}

Al FONACIT por financiar el proyecto interinstitucional "Caracterización de la biodiversidad de la cuenca del río Cucurital, afluente del río Caroní, estado Bolívar”, Agenda Biodiversidad, No 9803384 (Fundación de Ciencias Naturales La Salle, Fundación Instituto Botánico de Venezuela, Universidad de Los Andes, Universidad Simón Bolívar), del cual forma parte este trabajo. A Thalia Morales por su asistencia en el procesado y montaje de muestras botánicas. A Mayra García y Fernando García por su invalorable ayuda en el campo. A Carlos Varela por la identificación de algunas muestras. A Nataniel García, e Ernesto Flores por su valiosa colaboración como baquianos. Bruce Holst (SEL), Mauricio Ramia (VEN), Gustavo Romero (AMES), y Alan Smith (UC) amablemente identificaron muestras botánicas de Myrtaceae, Poaceae, Orchidaceae, y Pteridofitas, respectivamente.

\section{BIBLIOGRAFÍA CITADA}

Aymard, G.; Elcoro, S.; Marín, E.; Chaviel, A. 1997. Caracterización estructural y florística en bosques de tierra firme de un sector del bajo Río Caura, Estado Bolívar, Venezuela. In: Huber, O. \& Rosales, J. (Eds). Ecología de la Cuenca del Río Caura, Venezuela II. Estudios especiales. Sci. Guaianae, 7: 143-169.

Berry, P.E.; Holst, B.K.; Yatskievych, K. 1995a. Introduction. In: Steyermark, J.A., Berry, P.E.; Holst. B.K. (Eds). Flora of the Venezuelan Guayana. Volume 1: Introduction Missouri Botanical Garden, St. Louis; Timber Press, Portland.

Berry, P.E.; Huber, O.; Holst, B. 1995b. Floristic analysis and phytogeography. In: Steyermark, J.A., Berry, P.E.; Holst, B. (Eds). Flora of the Venezuelan Guayana. Volume 1: Introduction, pp 161-191. Missouri Botanical Garden, St. Louis; Timber Press, Portland.

Colonnello, G. 1999. Fisiografía y Geografía. In: Señaris, J. (Eds.). Caracterización de la diversidad biológica de la cuenca del río Cucurital, afluente del río Caroní, estado Bolívar, Venezuela. $1^{\circ}$ Informe técnico de avance proyecto Fonacit $N^{\circ}$ 98003384, pp 1-13. Museo de Historia Natural la Salle. Caracas, Venezuela.

Dezzeo, N.; Briceño, E. 1997. La vegetación de la cuenca del Río Chanaro: Medio Río Caura. In: Huber, O.; Rosales, J. (Eds). Ecología de la Cuenca del Río Caura, Venezuela II. Estudios especiales. Sci. Guaianae, 7: 365-385.

Hernández, L. 1999. Caracterización florística de los bosques. In: Hernández, L. (Ed). Ecología de la altiplanicie de la Gran Sabana (Guayana venezolana) II. Sci. Guaianae, 9: 53-83.

Huber, O. 1986. La vegetación de la cuenca del Río Caroní. Interciencia, 11: 301-310.

Huber, O. 1995a. Vegetation. In: Steyermark, J.A., Berry, P.E.; Holst, B.K. (Eds). Flora of the Venezuelan Guayana. Volume 1: Introduction, pp 97-160. Missouri Botanical Garden, St. Louis; Timber Press, Portland. 
Huber, O. 1995b. Geographycal and physical features. In: Steyermark, J.A., Berry, P.E. \& Holst, B. (eds). Flora of the Venezuelan Guayana. Volume 1: Introduction, pp 1-61. Missouri Botanical Garden, St. Louis; Timber Press, Portland.

Huber, O. 1995c. History of botanical explorations. In: Steyermark, J.A.; Berry, P.E.; Holst, B.K. (Eds.). Flora of the Venezuelan Guayana. Volume 1: Introduction, pp 63-95. Missouri Botanical Garden, St. Louis; Timber Press, Portland.

Knab-Vispo C.; Berry, P.E.; Rodríguez, G. 1999. Floristic and structural characterization of a lowland rain forest in the Lower Caura Watershed, Venezuelan Guayana. Acta Bot. Venez., 22(2): 325-359.

Ramírez, N.; Gil, C.; López, M.; Hokche, O.; Brito, I. 1988. Caracterización florística y estructural de una comunidad arbustiva en la Guayana venezolana (Gran Sabana, Edo. Bolívar). Acta Cient. Venez., 39: 457-469.
Salas, L., Berry, P.E.; Goldstein, I. 1997. Composición y estructura de una comunidad de árboles grandes en el valle del Río Tabaro, Venezuela: una muestra de 18,75 ha. In: Huber, O. \& J. Rosales, (Eds). Ecología de la Cuenca del Río Caura, Venezuela II. Estudios especiales. Sci. Guaianae, 7: 291-308.

Sioli, H. 1975. Tropical rivers as expresión of their terrestrial enviroments. In: Goley, F. and E. Medina (Eds) Tropical ecological systems. Trend and Aquatics Research. SpringerVerlag. New York.

Steyermark, J.A. 1966. Contribuciones a la flora de Venezuela: Flora del Ptari-Tepui. Acta Bot. Venez., 1: 30-104.

Steyermark, J.A., Berry, P.E.; Holst, B. (Gen. eds). 1995-2005. Flora of the Venezuelan Guayana. Volume 1-9: Missouri Botanical Garden, St. Louis.

Recebido em 31/03/2008

Aceito em 17/11/2008 